\title{
Which mechanism(s) for the $(d, p)$ reactions? Or can the CDCC approach be used to compute transfer cross sections?
}

\author{
Pierre Chau Huu-Tai ${ }^{1, a}$ \\ ${ }^{1}$ CEA,DAM,DIF, F-91297 Arpajon, France
}

\begin{abstract}
We propose to use the Continuum Discretized Coupled Channels approach to describe both the differential cross sections and the excitation fonctions for the $(\mathrm{d}, \mathrm{p})$ reactions.
\end{abstract}

\section{Introduction}

Just after the discovery of radioactivity, it was realized that nuclear reactions can be used to explore the structure of nuclei. In particular, since 1932 [1], the deuteron has been widely used as a projectile both to investigate the spectroscopic features of the compound nucleus and to produce isotopes relevant for technological or medical purposes. Indeed as a weakly bound two-nucleon system, this hydrogen isotope can easily transfer one of its component to the target nucleus ${ }_{Z}^{A} \mathrm{X}$ to form the compound nucleus ${ }_{Z}^{A+1} Y_{N+1}$ or ${ }_{Z+1}^{A+1} Y_{N}$ and as a charged particle, the incident energy can be accurately chosen allowing a selective study of the reaction. But, in spite of all these advantages and if many differential cross sections are quite well reproduced, the $(\mathrm{d}, \mathrm{p})$ or $(\mathrm{d}, \mathrm{n})$ excitation functions are often poorly described, meaning that our understanding of the reaction mechanism remains to be improved. It is due to the fact that, for these reactions, many processes (such as direct and multistep transfer, elastic and inelastic breakup...) can occur and will compete and interfere to produce the same compound nucleus. The cross section prediction thus requires to solve at least a quantum three-body problem which includes the continuum effects properly. In 1958, S. Watanabe [2] proposed to build the deuteron optical potential by folding the projectile wave function with the nucleon-target optical potentials in order to reproduce the elastic cross section. Then in the seventies, an improvement of this approach was developped: an approximated way to compute the deuteron elastic and elastic breakup cross sections was proposed by R.C. Johnson and P.J.R. Soper [3] (who used a simplified approach involving an adiabatic approximation) and by G.H. Rawitscher [4]. Within this model (now known as the Continuum Discretized Coupled Channels (CDCC) approach), the total wave function of the deuteron+target system is expanded into a sum of states describing the elastic channel and breakup ones and the cross sections for each channel are derived by solving a set of coupled differential equations. The CDCC has been widely studied and quite sucessfully applied, mainly to predict differential cross sections (see e.g. [5-10, 12-14]). Starting from some CDCC calculations, we will propose a semi-phenomenological method to compute the $(\mathrm{d}, \mathrm{p})$ excitation functions.

\footnotetext{
ae-mail: huu-tai.chau@cea.fr
} 


\section{A semi-phenomenological model to compute the excitation function using the CDCC approach}

\subsection{A brief preview of the CDCC philosophy}

The CDCC for deuteron induced reactions has been extended in order to also include the target excitations [11]. This extension will be denoted by $\mathrm{CDCC}^{*}$ and will be briefly summarized. Within this framework, the total wave function of the $\mathrm{d}+\operatorname{target}$ system depending on $\mathbf{R}$, the deuteron center of mass coordinates and on $\rho$, the proton-neutron relative coordinates, is written as:

$$
\left|\Psi_{J_{T} M_{T}}(\mathbf{R}, \rho)\right\rangle=\sum_{i l S I_{p} L J I_{t}}\left|(i l S) I_{p} L J I_{t} ; J_{T} M_{T}\right\rangle
$$

where the channels of the system for a given $J_{T}$ are labeled by the bin number $i$ ( $i=0$ for the deuteron ground state and $i>0$ for breakup ones), the deuteron spin $S=1$, the relative orbital angular momentum $l$, the angular momentum $J$, the orbital angular momentum $L$ associated to $\mathbf{R}$ and the spin of the target $I_{t}$ where $\vec{S}+\vec{l}=\overrightarrow{I_{p}}, \vec{L}+\overrightarrow{I_{p}}=\vec{J}$ and $\overrightarrow{I_{t}}+\vec{J}=\overrightarrow{J_{T}}$.

It satisfies the Schrödinger equation:

$$
\hat{H}\left|\Psi_{J_{T} M_{T}}(\mathbf{R}, \rho)\right\rangle=E\left|\Psi_{J_{T} M_{T}}(\mathbf{R}, \rho)\right\rangle
$$

After performing a multipole expansion and after left-multiplying the Schrödinger equation by $\langle c|$ and integrating over $\rho$ and the angular variables, the radial parts $u_{c}(R)$ of the wave function are solutions of a set of coupled differential equations:

$$
\left(-\frac{\hbar^{2}}{2 \mu_{R}} \frac{d^{2}}{d R^{2}}+\frac{\hbar^{2} L(L+1)}{2 \mu_{R} R^{2}}+V_{p}^{(\text {Coul })}-E_{i}\right) u_{c}(R)=-\sum_{c^{\prime}} V_{c c^{\prime}}(R) u_{c^{\prime}}(R)
$$

where $c$ and $c^{\prime}$ denote the channels $\left|(i l S) I_{p} L J I_{t} ; J_{T} M_{T}\right\rangle$ and $\left|\left(i^{\prime} l^{\prime} S^{\prime}\right) I_{p}^{\prime} L^{\prime} J^{\prime} I_{t}^{\prime} ; J_{T} M_{T}\right\rangle$ respectively. The expressions of the form factors $V_{c c^{\prime}}(R)=\left\langle c\left|V\left(\mathbf{r}_{i}\right)\right| c^{\prime}\right\rangle$ can be derived by using the Wigner-Eckart theorem: these form factors depend on some angular momentum couplings, on the target excitation, and on a folding of the nucleon-target optical potentials with the p-n wave functions.

The solutions of these equations are then used to compute the source term of the (d,p) reaction. The equation of this transfer reaction is then solved within the DWBA method i.e. it is assumed that the effect of the $(d, p)$ channel on the elastic and breakup ones is weak.

\subsection{Computation of the excitation functions}

For a given incident energy $E_{d}$, the cross section of any reaction involves three contributions

$$
\sigma\left(E_{d}\right)=\sigma_{\text {Direct }}\left(E_{d}\right)+\sigma_{\text {Pre-eq. }}\left(E_{d}\right)+\sigma_{\mathrm{CN}}\left(E_{d}\right)
$$

where $\sigma_{\text {Direct }}, \sigma_{\text {Preeq. }}$ and $\sigma_{\mathrm{CN}}$ are the direct component, the preequilibrium and the Compound Nucleus one, respectively. For the (d,p) cross section, the direct component reads

$$
\sigma_{\text {Direct }}^{(d, \text { nucleon })}\left(E_{d}\right)=\sum_{i \leq i_{\max }, l_{n}, j_{n}} \sigma_{l_{n}, j_{n}}\left(E_{x, i}, E_{d}\right)
$$

where $\sigma_{l_{n}, j_{n}}\left(E_{x, i}, E_{d}\right)$ denotes the cross section when the nucleon is captured by the target to form the $\mathrm{A}+1$ nucleus into a state whose excitation energy, orbital angular momentum and spin are $E_{x, i}, l_{n}, j_{n}$, 
respectively and where $i_{\max }$ depends on the Q-value of the reaction and on $E_{d}$. Therefore to compute the direct component, it is firstly necessary to know all the states that will be populated by the transfer and for each level, the associated cross section $\sigma_{l_{n}, j_{n}}\left(E_{x, i}, E_{d}\right)$ has to be computed which can be done by integrating the differential cross section:

$$
\sigma_{l_{n}, j_{n}}\left(E_{x, i}, E_{d}\right)=\int \frac{d \sigma_{l_{n}, j_{n}}}{d \Omega}\left(E_{x, i}, E_{d}\right) d \Omega .
$$

This differential cross section involves the wave function of the captured neutron which is usually derived by assuming that the level with excitation energy $E_{x, i}$ is a pure single-particle state and by using a Woods-Saxon potential whose depth is adjusted to get the correct excitation energy. To take into account that the state is not in fact a pure single-particle one, it is then necessary to introduce a spectroscopic factor, $S_{l_{n}, j_{n}, i}$, to renormalize the calculated cross section, which means that, for each level, the experimental cross section must be known at least for one incident energy:

$$
\sigma_{l_{n}, j_{n}}\left(E_{x, i}, E_{d}\right)=S_{l_{n}, j_{n}, i} \int \frac{d \sigma_{l_{n}, j_{n}}^{\text {calc. }}}{d \Omega}\left(E_{x, i}, E_{d}\right) d \Omega .
$$

Thus the total direct cross section reads:

$$
\sigma_{\text {Direct }}^{(d, \text { nucleon })}\left(E_{d}\right)=\sum_{i \leq i_{\max }, l_{n}, j_{n}} S_{l_{n}, j_{n}, i} \int \frac{d \sigma_{l_{n}, j_{n}}^{\text {calc. }}}{d \Omega}\left(E_{x, i}, E_{d}\right) d \Omega .
$$

However to do such calculation, one should overcome some difficulties. First, one should infer the levels that will be populated by the transfer reaction from the knowledge of the compound nucleus spectrum. Then, for each of these levels, one should be able to determine the spectroscopic factor which means that, at least, one experimental cross section has been measured. Unfortunetaly these assumptions are not always satisfied.

Therefore the direct component will be computed by using a simplified approach. Indeed, as it has been illustrated in [11], it has been observed that the shape of the excitation function of the integrated cross sections does not depend strongly on the level excitation energy but mainly on the orbital angular momentum, $l_{n}$. Thus it will be assumed that:

$$
\sigma_{l_{n}, j_{n}}^{\text {calc. }}\left(E_{x, i}, E_{d}\right) \propto \tilde{\sigma}_{l_{n}, j_{n}}\left(E_{d}\right),
$$

which means that the integrated cross sections can be written as the product of an amplitude depending on the excitation energy of the level, $A\left(E_{x, i}\right)$, times a function depending only on $l_{n}$ and on the incident energy:

$$
\sigma_{l_{n}, j_{n}}^{\text {calc. }}\left(E_{x, i}, E_{d}\right) \approx A\left(E_{x, i}\right) \tilde{\sigma}_{l_{n}, j_{n}}\left(E_{d}\right) .
$$

For each $l_{n}$, one can choose a level with a given excitation energy $E_{x, i_{0}}$ and the associated cross section $\sigma_{l_{n}, j_{n}, i_{0}}^{\text {calc }}\left(E_{d}\right)$ is then computed:

$$
\sigma_{l_{n}, j_{n}, i_{0}}\left(E_{d}\right) \equiv \sigma_{l_{n}, j_{n}}^{\text {calc. }}\left(E_{x, i_{0}}, E_{d}\right)=A\left(E_{x, i_{0}}\right) \tilde{\sigma}_{l_{n}, j_{n}}\left(E_{d}\right)
$$

meaning that

$$
\tilde{\sigma}_{l_{n}, j_{n}}\left(E_{d}\right)=\frac{1}{A\left(E_{x, i_{0}}\right)} \sigma_{l_{n}, j_{n}, i_{0}}\left(E_{d}\right)
$$

and that, for all excitation energies with the same $l_{n}$ :

$$
\sigma_{l_{n}, j_{n}}^{\text {calc. }}\left(E_{x, i}, E_{d}\right) \approx \frac{A\left(E_{x, i}\right)}{A\left(E_{x, i_{0}}\right)} \sigma_{l_{n}, j_{n}, i_{0}}\left(E_{d}\right) \text {. }
$$


After some transformations, it can be shown that the direct component of excitation function reads:

$$
\sigma_{\text {Direct }}^{(d, \text { nucleon })}\left(E_{d}\right) \approx \sum_{l_{n}, j_{n}}\left(\sum_{i \leq i_{\max }} S_{l_{n}, j_{n}, i} \frac{A\left(E_{x, i}\right)}{A\left(E_{x, i_{0}}\right)}\right) \sigma_{l_{n}, j_{n}, i_{0}}\left(E_{d}\right) .
$$

Denoting $\sum_{i \leq i_{\max }} S_{l_{n}, j_{n}, i} \frac{A\left(E_{x, i}\right)}{A\left(E_{x, i_{0}}\right)}=W_{l_{n}, j_{n}}\left(E_{d}\right)$, the direct contribution is therefore rewritten as:

$$
\sigma_{\text {Direct }}^{(d, \text { nucleon })}\left(E_{d}\right) \approx \sum_{l_{n}, j_{n}} W_{l_{n}, j_{n}}\left(E_{d}\right) \sigma_{l_{n}, j_{n}, i_{0}}\left(E_{d}\right)
$$

where the $W_{l_{n}, j_{n}}$ 's are non-decreasing bounded functions of $E_{d}$ thus one deduces that

$$
\exists \omega_{l_{n}, j_{n}}, \lim _{E_{d} \rightarrow \infty} W_{l_{n}, j_{n}}\left(E_{d}\right)=\omega_{l_{n}, j_{n}},
$$

and that:

$$
\sigma_{\text {Direct }}^{(d, \text { nucleon })}\left(E_{d}\right) \underset{\infty}{\sim} \sum_{l_{n}, j_{n}} \omega_{l_{n}, j_{n}} \sigma_{l_{n}, j_{n}, i_{0}}\left(E_{d}\right),
$$

which means that, at least for large incident energies, the excitation function can be approximated by a linear combination of the individual cross sections, $\sigma_{l_{n}, j_{n}, i_{0}}$.

In our approach, the computation of the direct component of the excitation function are performed within three steps:

- First, the individual cross sections $\sigma_{l_{n}, j_{n}, i_{0}}$ for a few given levels of the A+1 nucleus are calculated within the $\mathrm{CDCC}^{*}$-DWBA framework.

- Then the weights, $\omega_{l_{n}, j_{n}}$ are deduded by reproducing the experimental cross sections at some given incident energies.

- Using the expression (7), the direct part of the excitation function is obtained overall the incident energy range.

This semi-phenomenological approach will be illustrated in some details for ${ }^{75} \mathrm{As}$ in the subsection 2.3 and it will be compared with some experimental excitation functions for a large set of targets in the subsection 2.4

\subsection{Excitation function for ${ }^{75} \mathrm{As}$}

Using the $\mathrm{CDCC}^{*}$-DWBA approach , the individual $(\mathrm{d}, \mathrm{p})$ cross sections have been computed for three levels of ${ }^{76} \mathrm{As}$ : the ground state with $\left(l_{n}, j_{n}^{\pi}\right)=\left(4,7 / 2^{+}\right)$and two excited levels at $0.044 \mathrm{MeV}$ and $0.366 \mathrm{MeV}$ and with $\left(l_{n}, j_{n}^{\pi}\right)=\left(1,3 / 2^{-}\right),\left(l_{n}, j_{n}^{\pi}\right)=\left(3,5 / 2^{-}\right)$, respectively.

Some angular distributions of the ${ }^{75} \mathrm{As}(\mathrm{d}, \mathrm{p})^{76} \mathrm{As}$ reaction have been measured for deuteron incident at $12 \mathrm{MeV}$ by J.F. Lemming et al. [15] and by S. Mordechai et al. [16]. Since the neutron wave functions have not been computed for all the excited levels, when the excitation energy of the experimental level is different from the one used in the calculations, the comparison has be done with a "theoretical level" with the same quantum numbers. Some comparisons are plotted in Fig. 1. In these figures, the calculated differential cross sections and the experimental data are represented by the red curves and the black dots, respectively. An overall agreement between calculations and measurements can be observed. The shape of the excitation function computed for the three levels previously mentionned are plotted in arbitrary units in Fig. 2.

This excitation function has been measured by N. Baron et al. [17] at $E_{d}=19.0 \mathrm{MeV}$ and by H.F. Röhm et al. [18] for deuteron incident energies ranging from 18.7 to $51.3 \mathrm{MeV}$. These two sets 
of data are very different since the cross section measured by H.F. Röhm et al. at $18.7 \mathrm{MeV}$ is equal to $105 \pm 7.4 \mathrm{mb}$ while the one published by $\mathrm{N}$. Baron et al. is equal to $76.9 \pm 11.5 \mathrm{mb}$. $>$ From the shape of the data of H.F. Röhm et al., we have deduced that the calculated cross section with $l_{n}=1$ is the main contribution and we have extracted the corresponding weights. The comparison between the calculation and the data is represented in the right panel of the Fig. 2.

\subsection{Comparison with some experimental data}

Similar calcutions have been performed for nuclei ranging from Al to Hf. A more detailled description of the ingredients used for each nucleus is given in [11]. The comparisons with the experimental data [19-36] are plotted in Fig. 3-14. Three conclusions can be drawn from these comparisons:

- A quite good agreement is obtained for all these nuclei.

- In most of the cases, the contribution of only one excitation function with a given orbital angular momentum $l_{n}$ is needed to reproduce the experimental data, the value of $l_{n}$ depending on the neutron number of the target.

- It is amazing to get such an agreeemnt within the whole energy range since one would expect that the compound nucleus and the pre-equilibrium processes would play a role in the reaction mechanism.
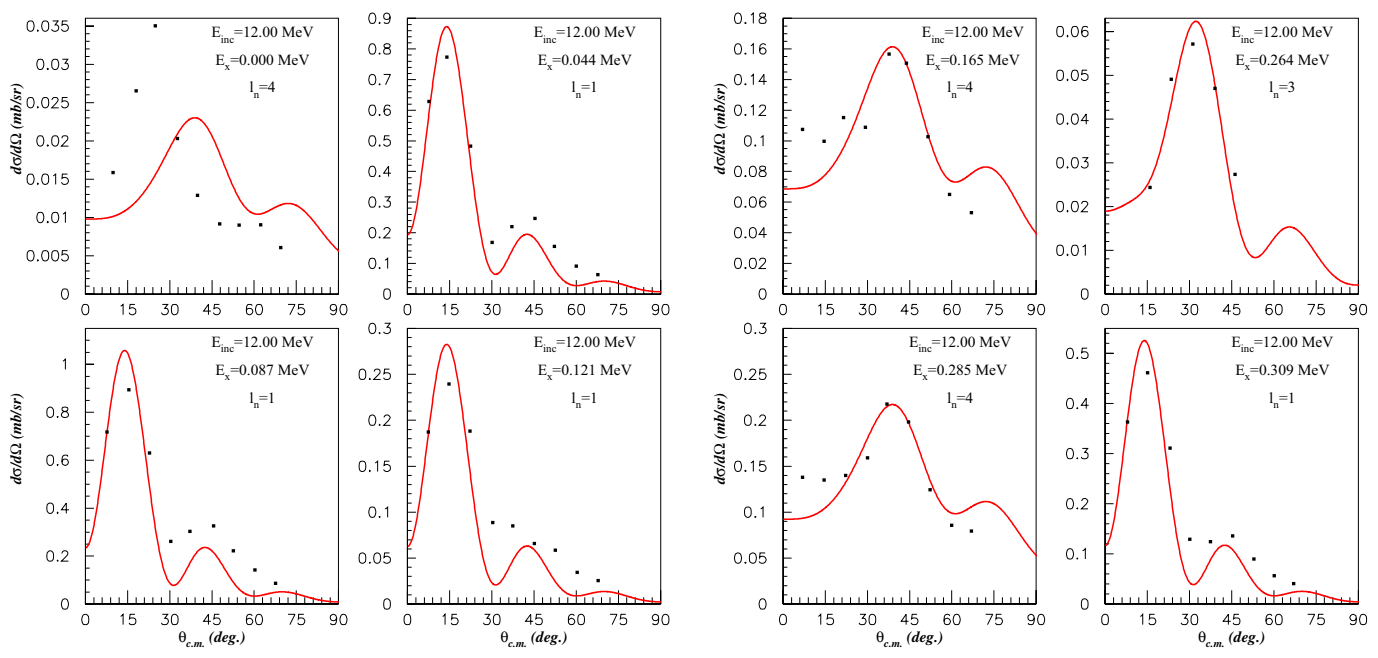

Figure 1. Differential cross sections for the ${ }^{75} \mathrm{As}(\mathrm{d}, \mathrm{p})$ reaction. The experimental data (dots) are from [15].

\section{Conclusion}

In order to get a better description of differential and integral cross sections for the (d,p) reaction, a semi-phenomenological approach has been built. Its starting point are some CDCC*-DWBA calculations of the individual cross sections for a restricted number of states of the compound nucleus since it has been numerically observed that the shape of the integrated cross section as function of the incident energy is almost independent of the excitation energy of the compound nuleus state. It is then shown 

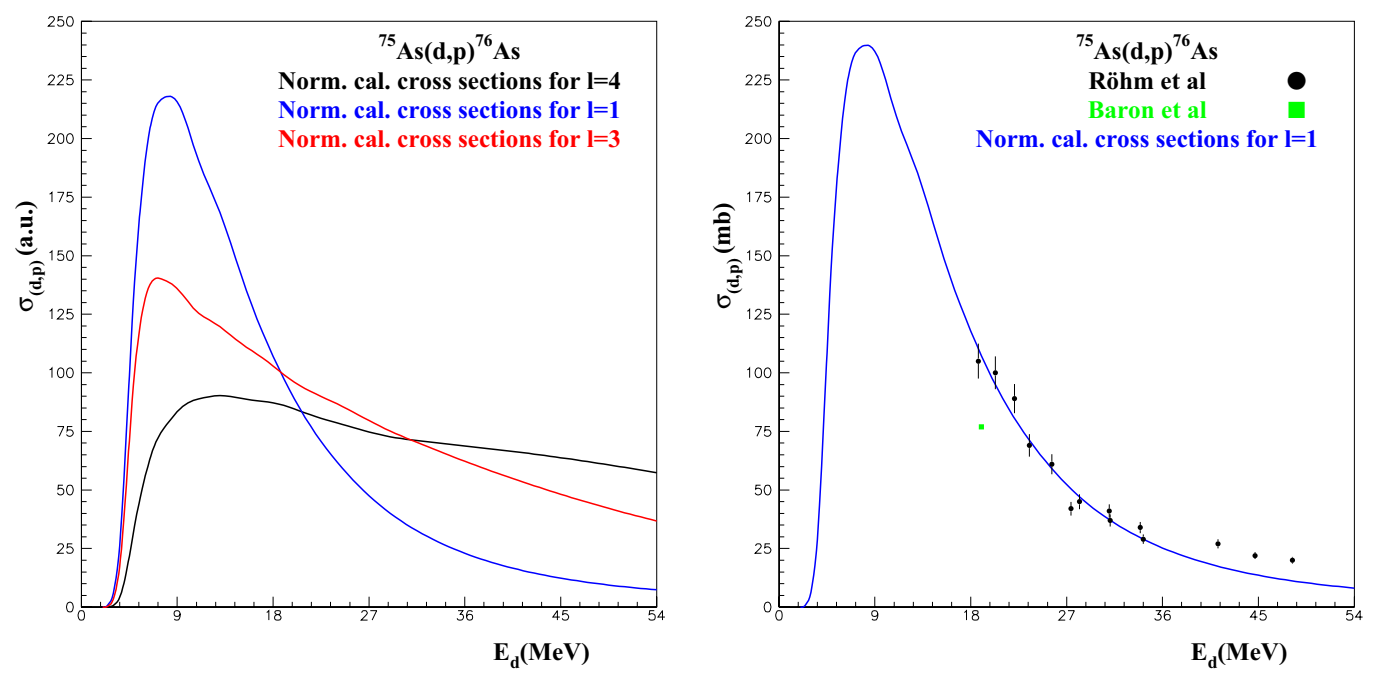

Figure 2. Left panel: integrated cross sections in arbitrary unit for the three levels of ${ }^{76} \mathrm{As}$ defined in the text. Right panel: comparison between the ${ }^{75} \mathrm{As}(\mathrm{d}, \mathrm{p})^{76} \mathrm{As}$ experimental excitation function $[17,18]$ and a linear combination of the theoretical excitation functions as defined by Eq. (7).

that the direct part of the excitation function can be written as a linear combination of these individual excitation functions. A quite good agreement between the experimental excitation functions and the calculated ones has been obtained for a large number of nuclei. But despite this agreement, many open questions remain to be answered, in particular it is not clear why there is no room left for other reaction mechanisms such as compound nucleus or pre-equilibrium processes.

\section{References}

[1] H.C. Urey, F.G. Brickwedde, G.M. Murphy, Phys. Rev. 39, 164 (1932).

[2] S. Watanabe, Nucl. Phys. 8, 484 (1958).

[3] R.C. Johnson, P.J.R. Soper, Phys. Rev. C 1, 976 (1970).

[4] G.H. Rawitscher, Phys. Rev. C 9, 2210 (1974).

[5] M. Yahiro, M. Kamimura, Prog. Theor. Phys. 65, 2046 (1981).

[6] M. Yahiro, M. Kamimura, Prog. Theor. Phys. 65, 2051 (1981).

[7] M. Yahiro et al., Prog. Theor. Phys. 67, 1467 (1982).

[8] M. Yahiro et al., Prog. Theor. Phys. Suppl. 89, 1 (1986).

[9] N. Austern et al., Phys. Rep. 154, 125 (1987).

[10] P. Chau-Huu Tai, Nucl. Phys. A 773, 56 (2006).

[11] P. Chau-Huu Tai, Eur. Phys. J. A 51, 166 (2015).

[12] M. Masaki et al., Nucl. Phys. A 573, 1 (1994).

[13] K. Hirota et al., Nucl. Phys. A 628, 547 (1998).

[14] M. Yamagushi et al., Nucl. Phys. A 747, 3 (2005).

[15] J.F. Lemming et al., Nucl. Phys. A 239412 (1975). 

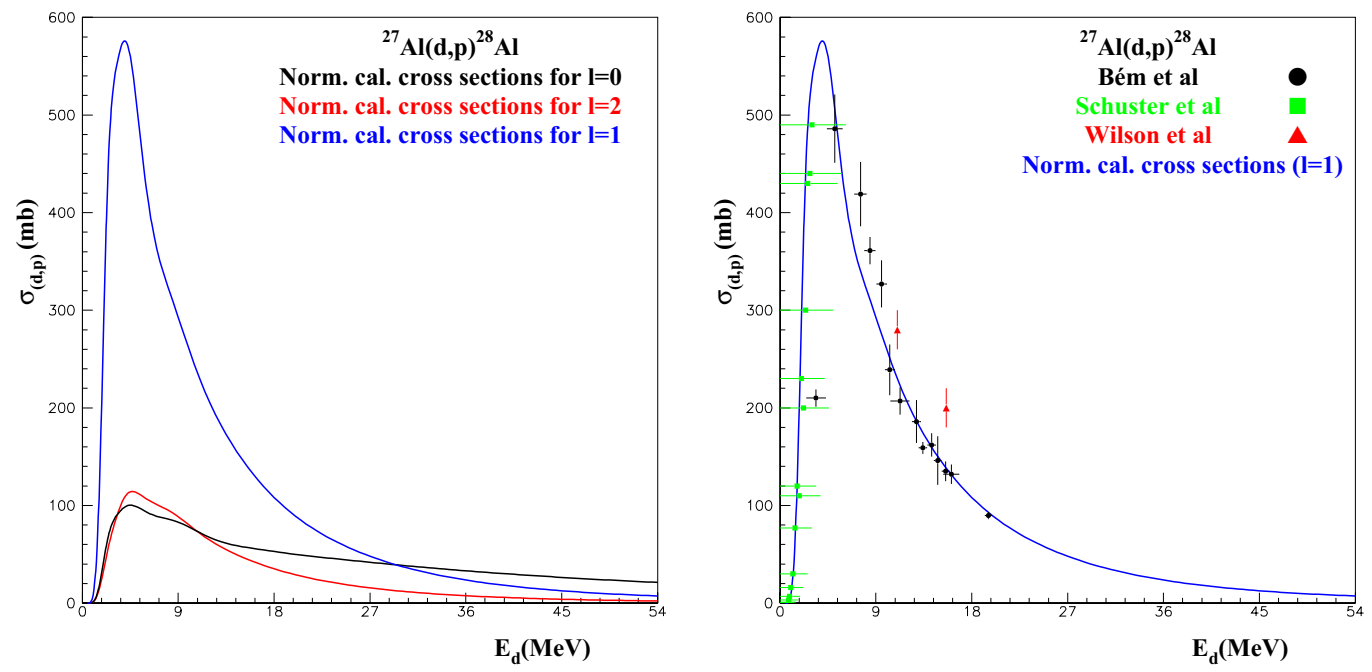

Figure 3. Left panel: integrated cross sections in arbitrary unit for three levels of ${ }^{28} \mathrm{Al}$. Right panel: comparison between the ${ }^{27} \mathrm{As}(\mathrm{d}, \mathrm{p})^{28} \mathrm{Al}$ experimental excitation function [19-21] and a linear combination of the theoretical excitation functions as defined by Eq. (7).

[16] S. Mordechai et al., Nucl. Phys. A 27282 (1976).

[17] N. Baron, B.L. Cohen, Phys. Rev. 129, 2636 (1963) doi: 10.1103/PhysRev.129.2636.

[18] H.F. Röhm, H. Münzel, J. Inorg. Nucl. Chem. 34,1773 (1972) doi: 10.1016/00221902(72)80523-2.

[19] E. Schuster, K. Wohlleben, Int. J. Appl. Radiat. Isot. 19471 (1968) doi: 10.1016/0020708X(68)90100-2.

[20] R.L. Wilson et al., Phys. Rev. C 13, 976 (1976) doi: 10.1103/PhysRevC.13.976.

[21] P. Bém et al., Phys. Rev. C 79, 044610 (2009) doi: 10.1103/PhysRevC.79.044610.

[22] L. Závorka et al., J. Korean Phys. Soc. 59, 1961 (2011) doi: 10.3938/jkps.59.1961.

[23] M.U. Khandaker et al., Nucl. Instrum. Methods B 316, 33 (2013) doi: 10.1016/j.nimb.2013.08.032.

[24] B. Kiraly et al., Nucl. Instrum. Methods B 267, 15 (2009) doi: 10.1016/j.nimb.2008.11.005.

[25] F. Ditrói et al., Nucl. Instrum. Methods B 270, 61 (2012) doi: 10.1016/j.nimb.2011.10.010.

[26] R. Michel, M. Galas, Int. J. Appl. Radiat. Isot. 34, 1325 (1983) doi: 10.1016/0020708X(83)90023-6.

[27] F. Ditrói et al., Nucl. Instrum. Methods B 268, 2571 (2010) doi: 10.1016/j.nimb.2010.06.033.

[28] H.I. West Jr et al., Phys. Rev. C 47, 248 (1993) doi: 10.1103/PhysRevC.47.248.

[29] F. Ditrói et al., Appl. Radiat. Isot. 70, 574 (2012) doi: 10.1016/j.apradiso.2011.12.034.

[30] F. Simonelli et al., Radiochim. Acta 98, 187 (2010) doi: 10.1524/ract.2010.1709.

[31] F. Tárkányi et al., Nucl. Instrum. Methods B 316, 22 (2013) doi: 10.1016/j.nimb.2013.08.031.

[32] J. Lange, H. Münzel, Radiochim. Acta 9, 66 (1968) doi: 10.1524/ract.1968.9.23.66.

[33] A. Hermanne et al., Nucl. Instrum. Methods B 267, 727 (2009) doi: 10.1016/j.nimb.2008.12.017.

[34] F. Tárkányi et al., Nucl. Instrum. Methods B 266, 3529 (2008) doi: 10.1016/j.nimb.2008.05.123. 

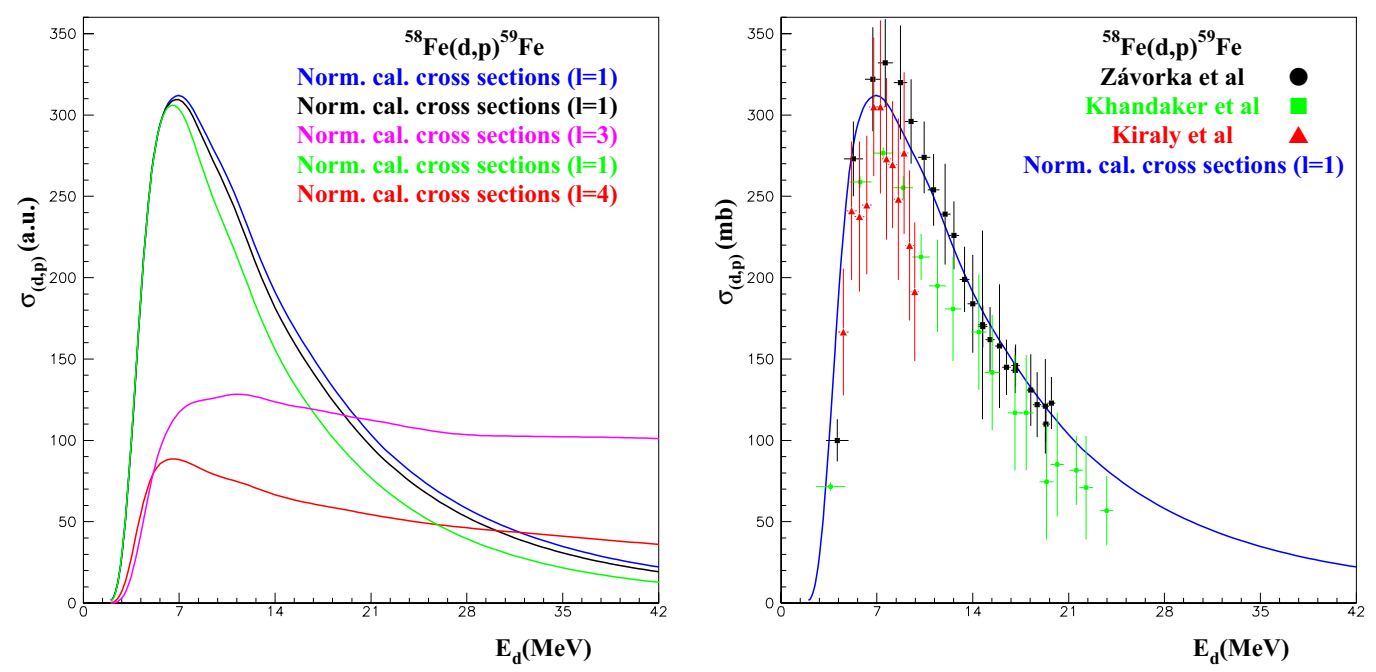

Figure 4. Left panel: integrated cross sections in arbitrary unit for five levels of ${ }^{58} \mathrm{Fe}$. Right panel: comparison between the ${ }^{58} \mathrm{Fe}(\mathrm{d}, \mathrm{p}){ }^{59} \mathrm{Fe}$ experimental excitation function [22-25] and a linear combination of the theoretical excitation functions as defined by Eq. (7).

[35] A. Hermanne et al., Nucl. Instrum. Methods B 311, 102 (2013) doi: 10.1016/j.nimb.2013.06.014. [36] S. Takács et al., Nucl. Instrum. Methods B 268, 3443 (2010) doi: 10.1016/j.nimb.2010.08.016. 

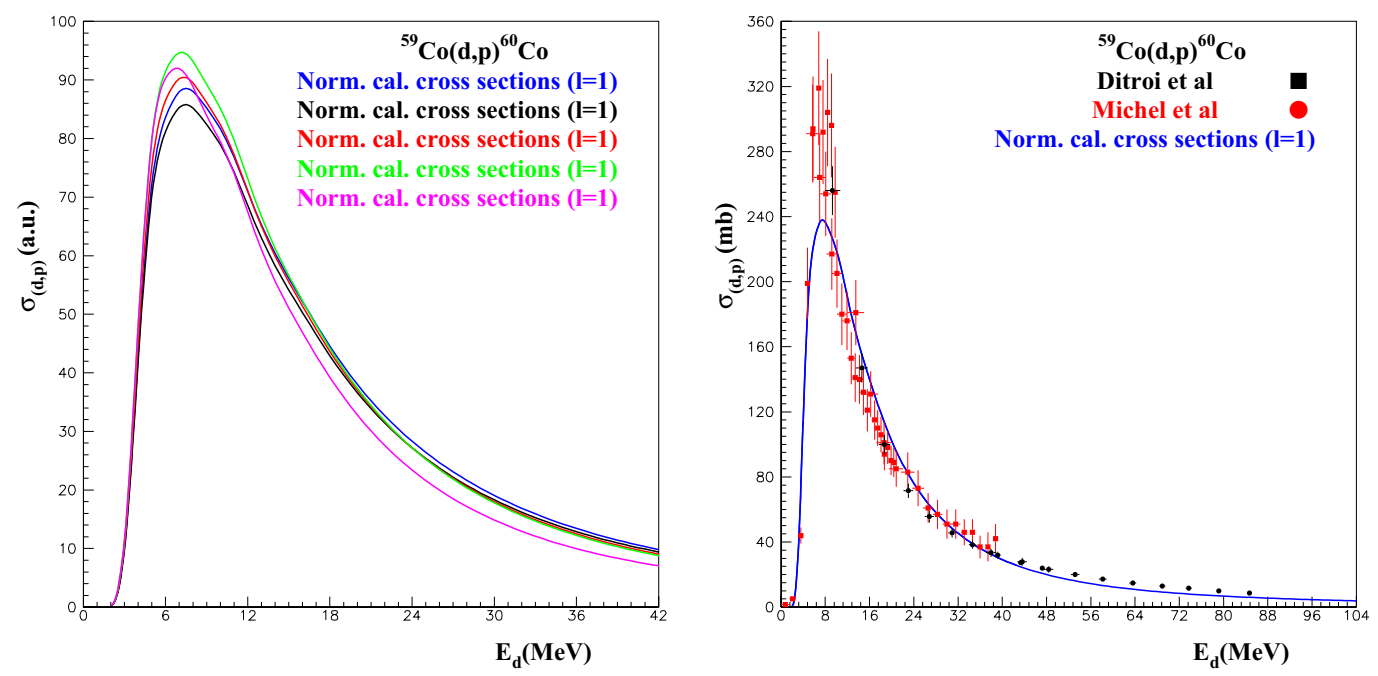

Figure 5. Left panel: integrated cross sections in arbitrary unit for five levels of ${ }^{59} \mathrm{Co}$. Right panel: comparison between the ${ }^{59} \mathrm{Co}(\mathrm{d}, \mathrm{p}){ }^{60} \mathrm{Co}$ experimental excitation function $[26,27]$ and a linear combination of the theoretical excitation functions as defined by Eq. (7).
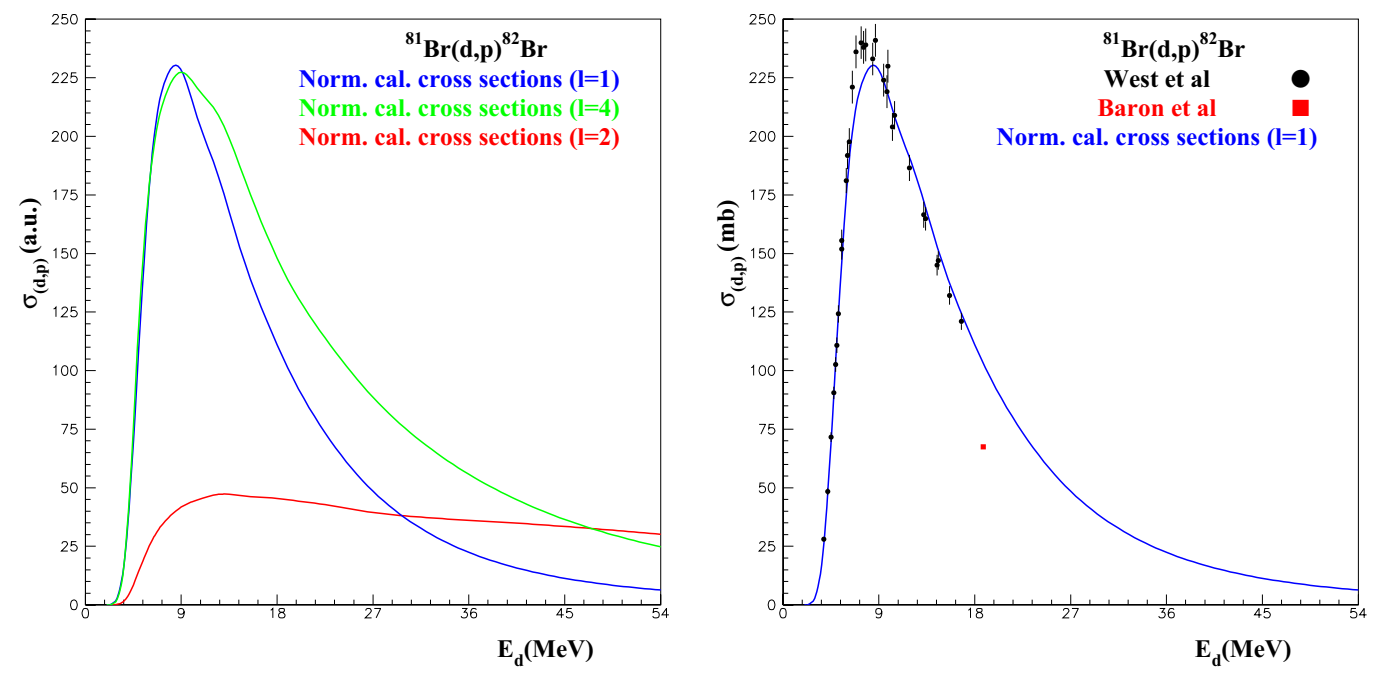

Figure 6. Left panel: integrated cross sections in arbitrary unit for three levels of ${ }^{81} \mathrm{Br}$. Right panel: comparison between the ${ }^{81} \mathrm{Br}(\mathrm{d}, \mathrm{p})^{82} \mathrm{Br}$ experimental excitation function $[17,28]$ and a linear combination of the theoretical excitation functions as defined by Eq. (7). 

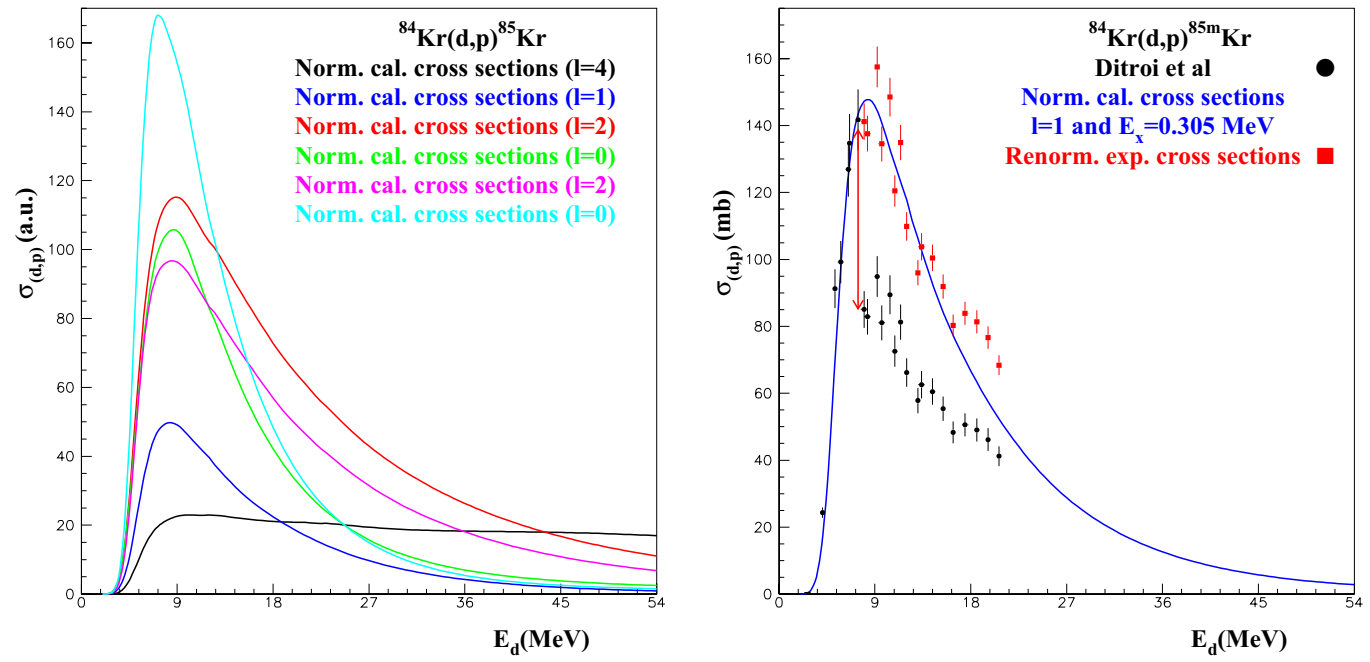

Figure 7. Left panel: integrated cross sections in arbitrary unit for six levels of ${ }^{84} \mathrm{Kr}$. Right panel: comparison between the ${ }^{84} \mathrm{Kr}(\mathrm{d}, \mathrm{p})^{85 m} \mathrm{Kr}$ experimental excitation function [29] and a linear combination of the theoretical excitation functions as defined by Eq. (7).
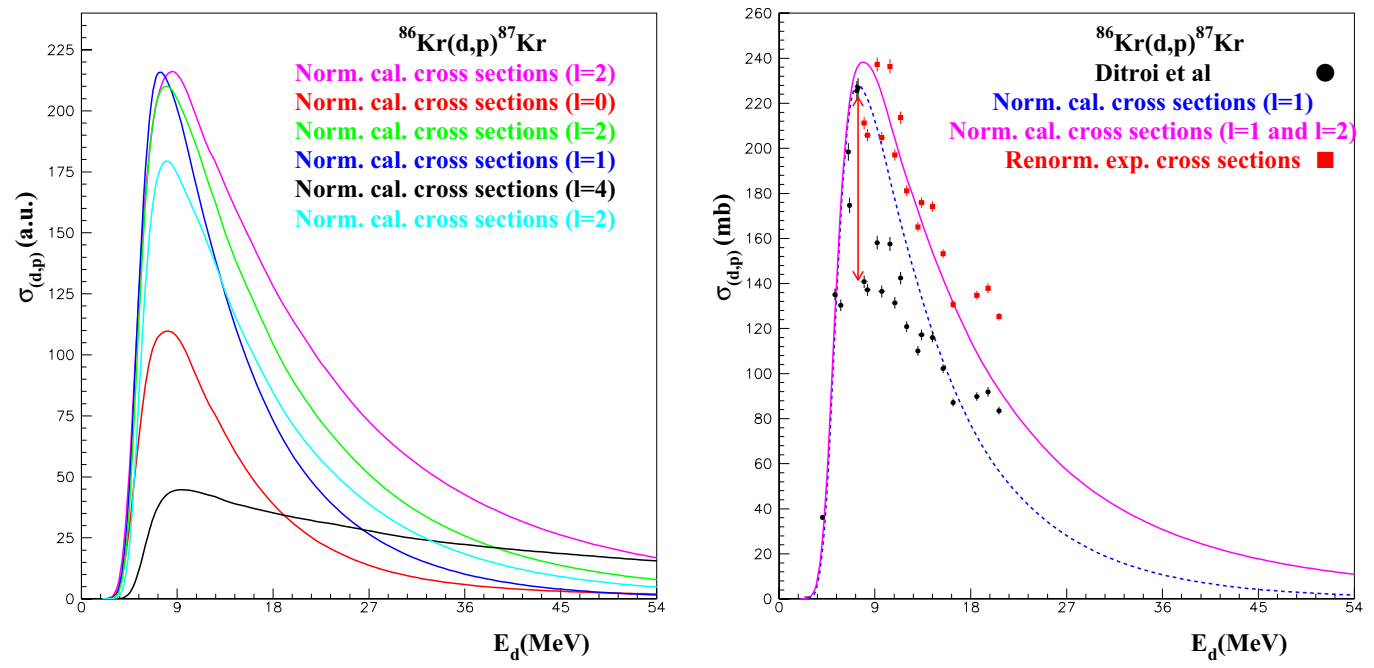

Figure 8. Left panel: integrated cross sections in arbitrary unit for six levels of ${ }^{86} \mathrm{Kr}$. Right panel: comparison between the ${ }^{86} \mathrm{Kr}(\mathrm{d}, \mathrm{p}){ }^{87} \mathrm{Kr}$ experimental excitation function [29] and a linear combination of the theoretical excitation functions as defined by Eq. (7). 

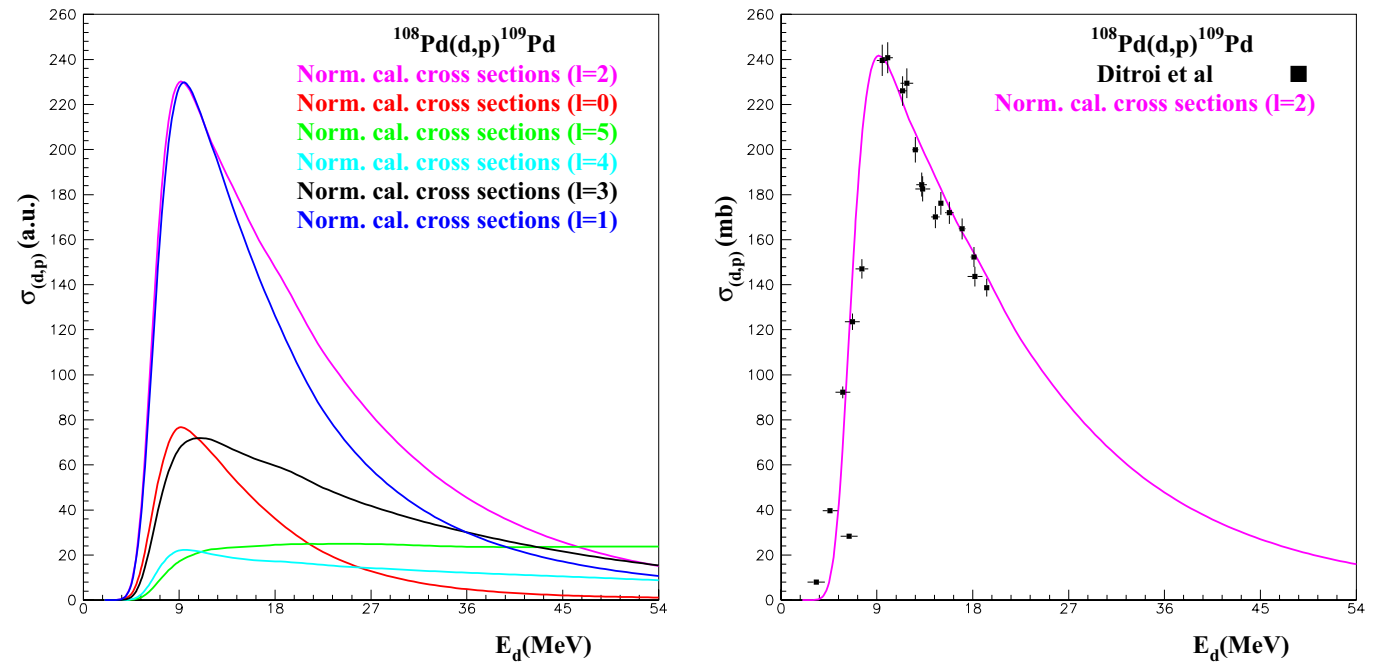

Figure 9. Left panel: integrated cross sections in arbitrary unit for six levels of ${ }^{180} \mathrm{Pd}$. Right panel: comparison between the ${ }^{108} \mathrm{Pd}(\mathrm{d}, \mathrm{p})^{109} \mathrm{Pd}$ experimental excitation function [25] and a linear combination of the theoretical excitation functions as defined by Eq. (7).
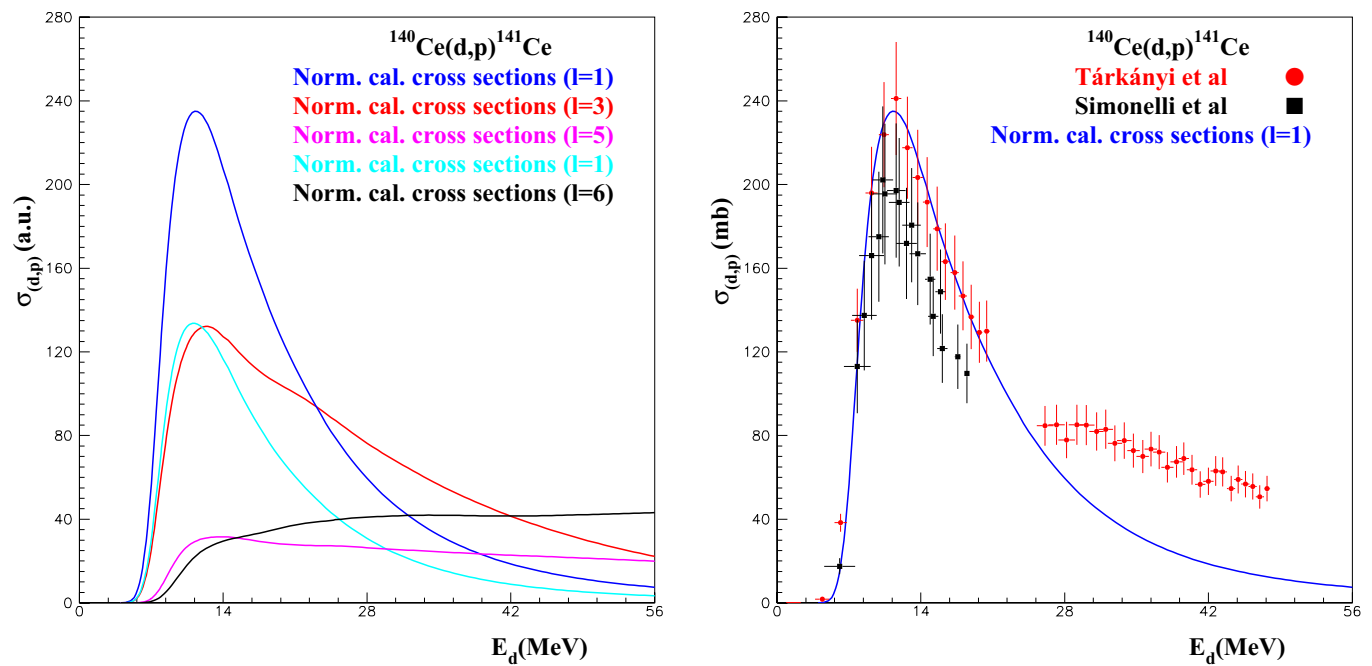

Figure 10. Left panel: integrated cross sections in arbitrary unit for five levels of ${ }^{140} \mathrm{Ce}$. Right panel: comparison between the ${ }^{140} \mathrm{Ce}(\mathrm{d}, \mathrm{p}){ }^{141} \mathrm{Ce}$ experimental excitation function $[30,31]$ and a linear combination of the theoretical excitation functions as defined by Eq. (7). 

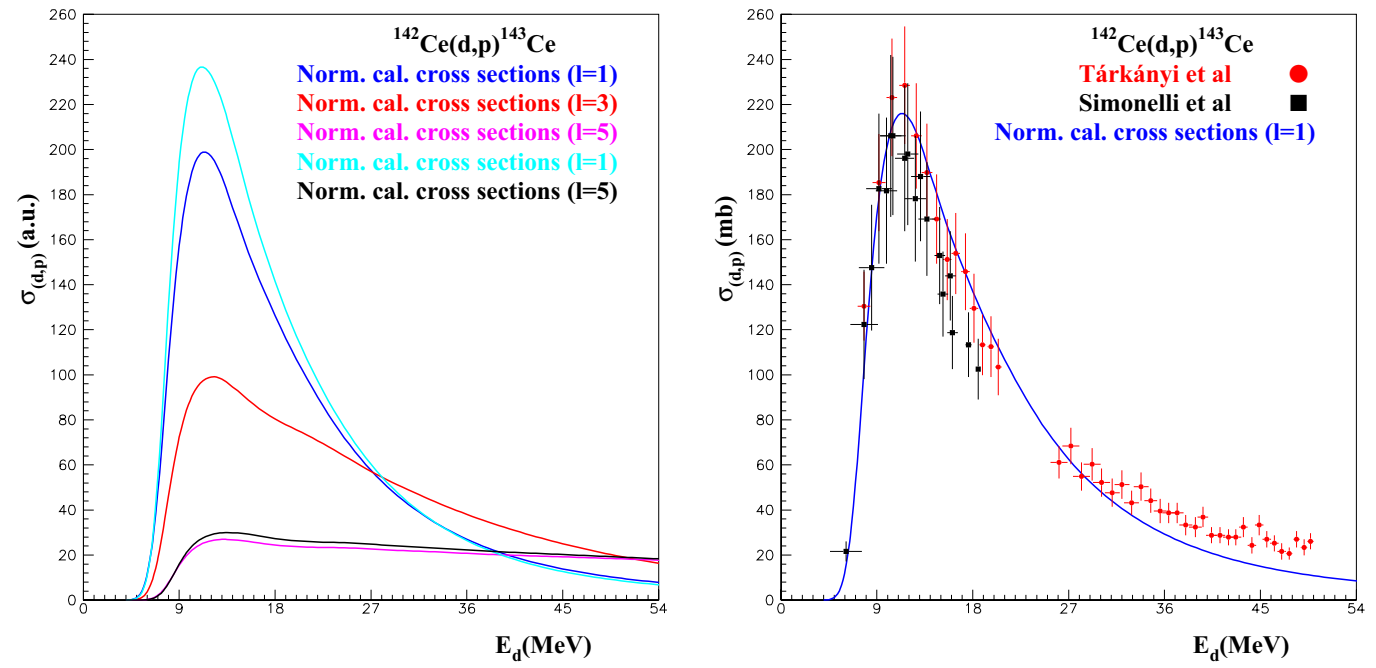

Figure 11. Left panel: integrated cross sections in arbitrary unit for five levels of ${ }^{142} \mathrm{Ce}$. Right panel: comparison between the ${ }^{142} \mathrm{Ce}(\mathrm{d}, \mathrm{p}){ }^{143} \mathrm{Ce}$ experimental excitation function $[30,31]$ and a linear combination of the theoretical excitation functions as defined by Eq. (7).
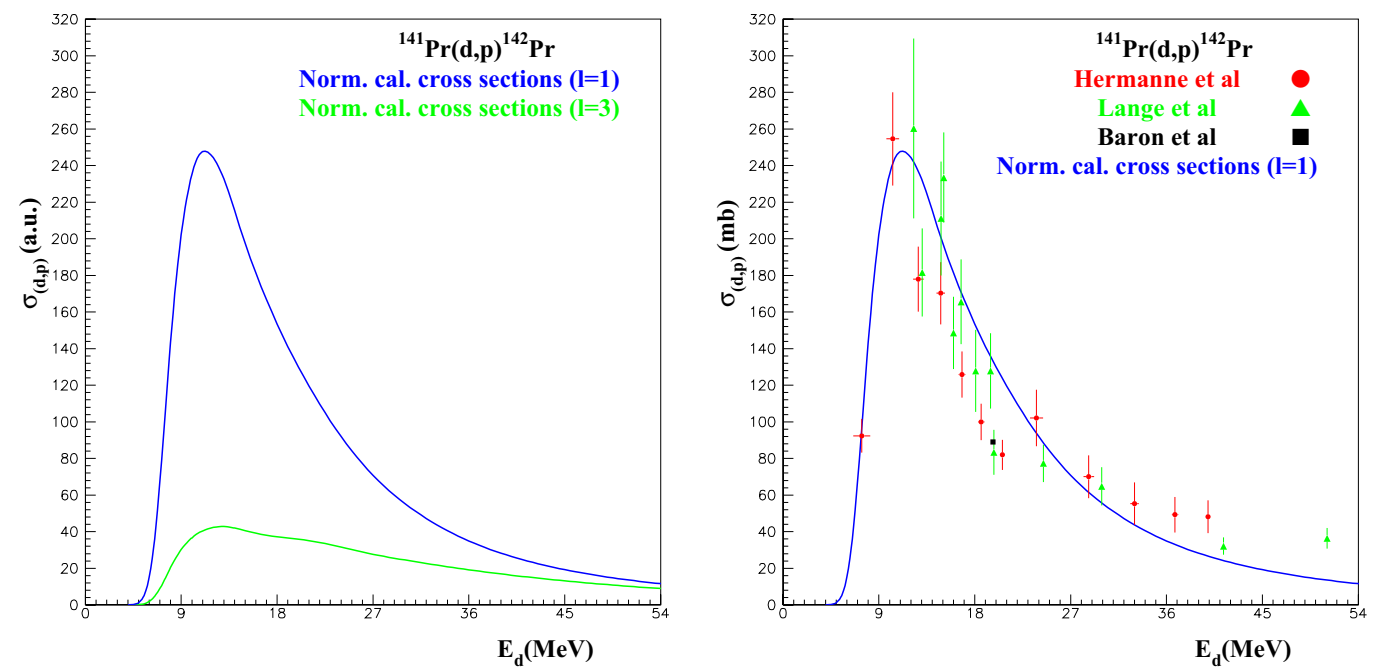

Figure 12. Left panel: integrated cross sections in arbitrary unit for two levels of ${ }^{141} \mathrm{Pr}$. Right panel: comparison between the ${ }^{141} \operatorname{Pr}(\mathrm{d}, \mathrm{p}){ }^{142} \operatorname{Pr}$ experimental excitation function [17, 32, 33] and a linear combination of the theoretical excitation functions as defined by Eq. (7). 

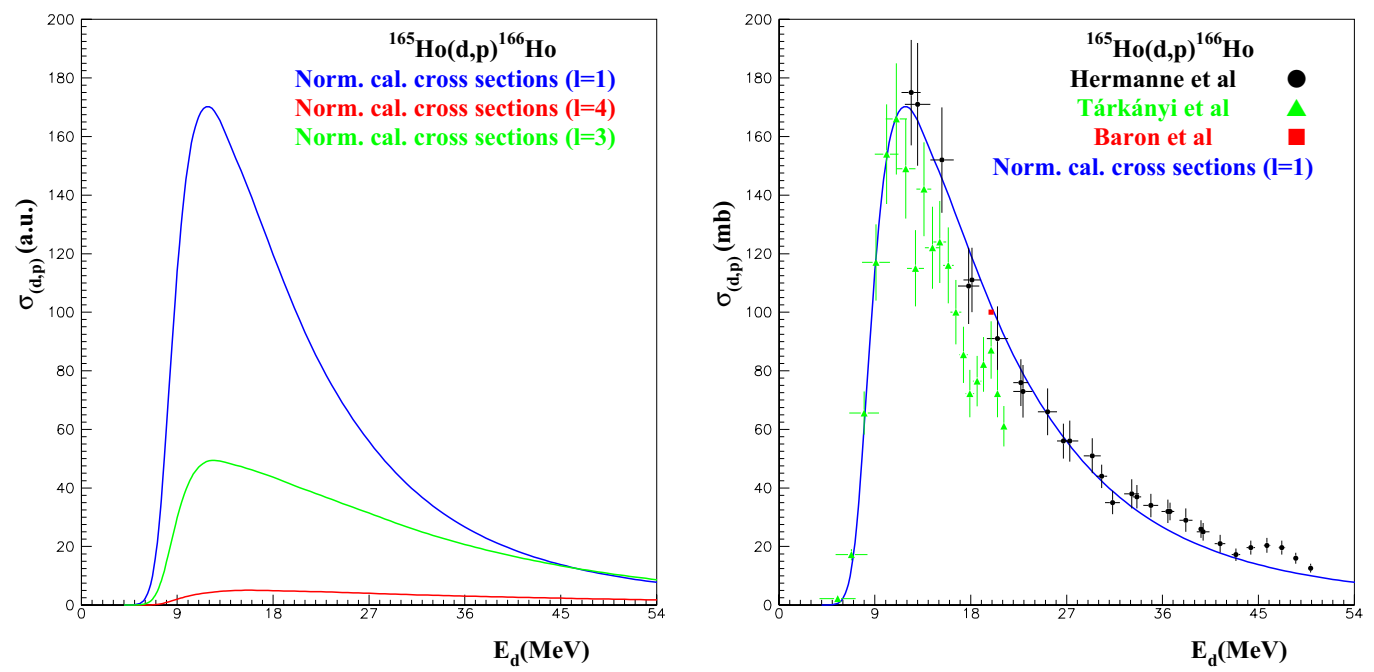

Figure 13. Left panel: integrated cross sections in arbitrary unit for three levels of ${ }^{165} \mathrm{Ho}$. Right panel: comparison between the ${ }^{165} \mathrm{Ho}(\mathrm{d}, \mathrm{p})^{166} \mathrm{Ho}$ experimental excitation function $[17,34,35]$ and a linear combination of the theoretical excitation functions as defined by Eq. (7).
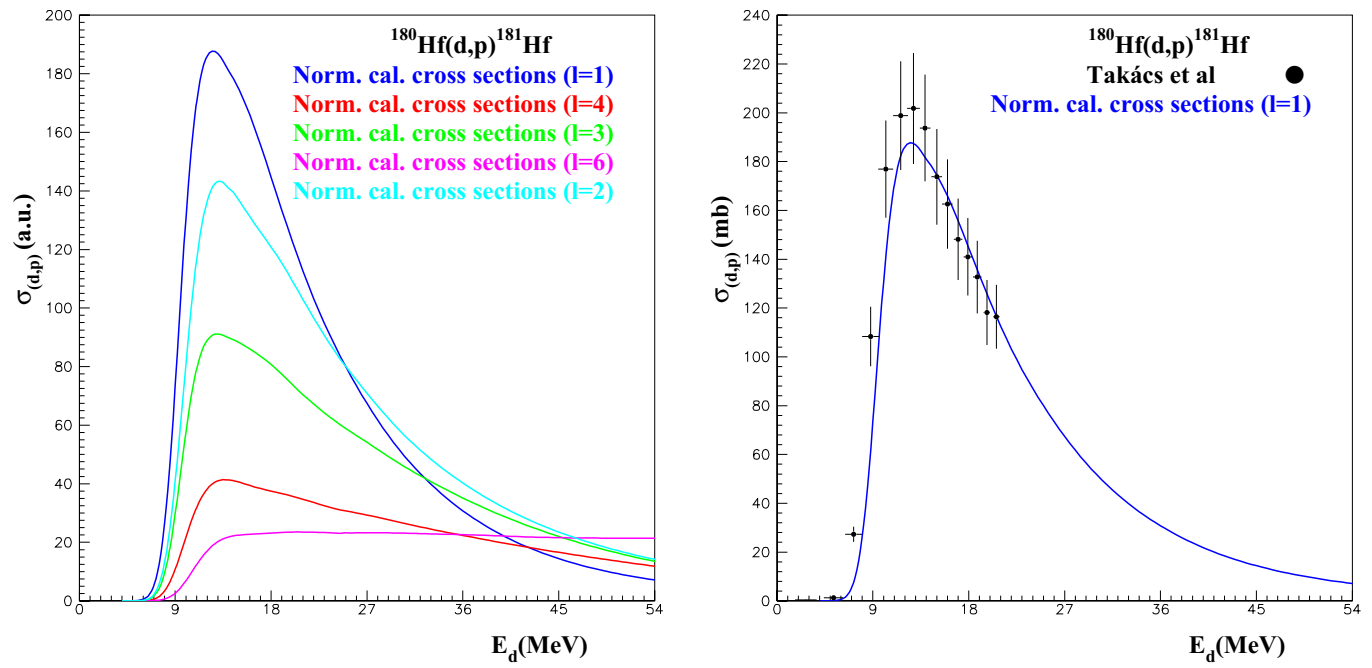

Figure 14. Left panel: integrated cross sections in arbitrary unit for five levels of ${ }^{181} \mathrm{Hf}$. Right panel: comparison between the ${ }^{180} \mathrm{Hf}(\mathrm{d}, \mathrm{p})^{181} \mathrm{Hf}$ experimental excitation function [36] and a linear combination of the theoretical excitation functions as defined by Eq. (7). 